krankhafte Befunde zahlenmäßig hoch, aber pathophysiologisch meist unbedeutend (z. B. alterstypische Klappenveränderungen). Eine linksventrikuläre Auswurffraktion unter $45 \%$ hatten nur $6 \%$ der Probanden.

Martinez-Sellés $M$ et al. Centenarians and their hearts: A prospective registry with comprehensive geriatric assessment, electrocardiogram, echocardiography, and follow-up. Am Heart J 2015; 169(6): 798-805

\section{Kommentar}

In der Population der 100-Jährigen führen kardiovaskuläre Ursachen am häufigsten zum Tod. Trotzdem weiß man wenig über die anatomischen, physiologischen und pathophysiologischen Befunde in dieser Altersgruppe, die in den letzten Jahrzehn- ten zwar prozentual deutlich gewachsen ist, aber quantitativ klein bleibt und nur selten Thema wissenschaftlicher Publikationen ist. Einerseits kommt es im Alter zu erheblichen strukturellen und funktionellen kardiovaskulären Änderungen, andererseits handelt es sich bei den 100-Jährigen um eine Selektion von Personen mit privilegierter Gesundheit mit langer Lebenserwartung.

Die Probanden beurteilten ihren Gesundheitsstatus überwiegend als gut: Auf einer Skala von 1 (sehr schlecht) bis 10 (sehr gut) mit 8,3 bei fehlenden bzw. 6,8 bei nachweisbaren EKG-Befunden. Eine Auswurffraktion unter $50 \%$ und Klappenveränderungen machten keinen Unterschied in der subjektiven Befindlichkeit. Viele haben einen gesunden Lebensstil geführt (körperliche Aktivität auch nach dem 65. Lebensjahr,
Nichtraucher und Alkoholverzicht). Insgesamt gewinnt man den Eindruck, dass Senioren über 100 Jahren sehr viel stärker von Abhängigkeit, Mangelernährung und Hirnleistungsstörungen beeinträchtigt sind als durch kardiale Beschwerden.

Die Senioren dieser Studie haben offenbar das Problem des Alterns gemeistert: Sie haben die altersbedingte Abnahme der Leistungsfähigkeit akzeptiert und den eigenen Anspruch reduziert.

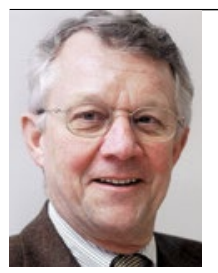

Prof. Dr. med. Heinrich Holzgreve Kardiogische Praxis, München

\title{
Lungenembolie durch CT überdiagnostiziert?
}

\section{Lungenembolien werden klinisch einerseits massiv unterdiagnostiziert, andererseits aber auch in einigen wenigen Fällen durch den Einsatz der thorakalen Computertomografie überdiagnostiziert.}

$\mathrm{N}$ ach wie vor ist die Lungenembolie unterdiagnostiziert. $\mathrm{Zu}$ unspezifisch sind die Symptome und die klinischen Zeichen. Hinzu kommt, dass im Fall eines Todes aufgrund einer Lungenembolie die eigentliche Todesursache häufig falsch eingeschätzt wird und viele Fälle einer akuten Lungenembolie als akuter Herzinfarkt fehlgedeutet werden.

Bei den apparativen diagnostischen Verfahren hat die hochauflösende Computertomografie (Multidetektor-CT) einen wichtigen Stellenwert, insbesondere in Kombination mit der transthorakalen Echokardiografie. Doch eine aktuelle Studie ergab nun, dass die Lungenembolie durch den diagnostischen Einsatz einer CT überdiagnostiziert werden kann. Man findet dabei zu viele kleine, ungefährliche Emboli, die jedoch nicht auffällig geworden wären, wenn man nicht nach ihnen gesucht hätte.

Im Rahmen eines Reviews wurden die CT-Aufnahmen von 175 Patienten, bei denen damit eine akute Lungenembolie diagnostiziert wurde, von einem Exper-

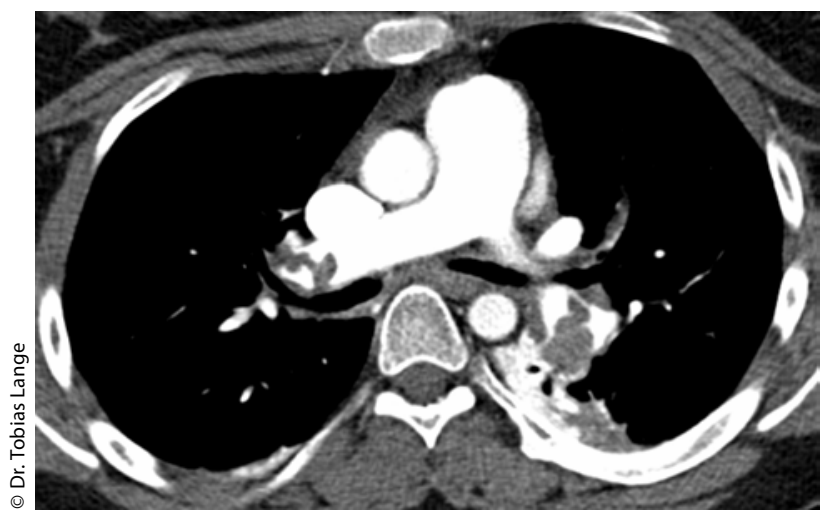

Akute Lungenembolie.

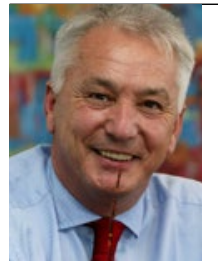

Prof. Dr. med. Curt Diehm

Max-Grundig-Klinik, Bühlerhöhe/Baden Baden 